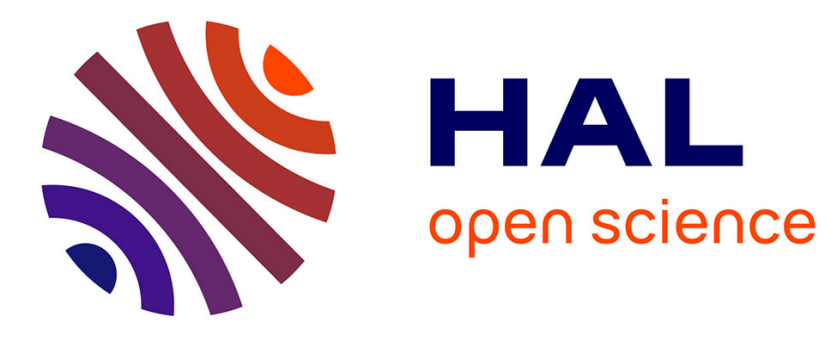

\title{
La Bible et le républicanisme selon John Milton
}

Christophe Tournu

\section{To cite this version:}

Christophe Tournu. La Bible et le républicanisme selon John Milton. Bulletin de la Société d'Etudes Anglo-Américaines des XVIIème et XVIIIème siècles, 1997, 44 (1), pp.147-164. 10.3406/xvii.1997.1372 . hal-03135387

\section{HAL Id: hal-03135387 https://hal.science/hal-03135387}

Submitted on 8 Feb 2021

HAL is a multi-disciplinary open access archive for the deposit and dissemination of scientific research documents, whether they are published or not. The documents may come from teaching and research institutions in France or abroad, or from public or private research centers.
L'archive ouverte pluridisciplinaire HAL, est destinée au dépôt et à la diffusion de documents scientifiques de niveau recherche, publiés ou non, émanant des établissements d'enseignement et de recherche français ou étrangers, des laboratoires publics ou privés. 


\section{La Bible et le républicanisme selon John Milton}

Christophe Tournu

\section{Citer ce document / Cite this document :}

Tournu Christophe. La Bible et le républicanisme selon John Milton. In: XVII-XVIII. Bulletin de la société d'études angloaméricaines des XVIle et XVIIle siècles. N44, 1997. pp. 147-164;

doi : https://doi.org/10.3406/xvii.1997.1372

https://www.persee.fr/doc/xvii_0291-3798_1997_num_44_1_1372

Fichier pdf généré le 28/03/2018 


\section{LA BIBLE ET LE RÉPUBLICANISME SELON JOHN MILTON ${ }^{1}$}

S'il est persuadé que la Bible prescrit une discipline ecclésiale adéquate. John Milton, en 164l-1642, ne pense pas qu'il faille encore y chercher une forme spécifique de gouvernement civil - Christ ne se mêle pas de politique. Cependant, en 1659-1660, alors que l'Angleterre sombre dans "this distracted anarchy," le pamphlétaire, repoussé dans ses derniers retranchements, doit s'y résoudre. ${ }^{2}$ Sur une base scripturaire, il préconise une république comparable à celle de 1649-1653: il fallait que deux conditions fussent réunies pour qu'elle voie le jour: le pouvoir et la puissance ne seraient pas détenus par un seul homme; la fonction de chef de l'État ne pourrait pas être héréditaire. Troisième exigence, selon Milton: le gouvernement civil ne devrait pas intervenir dans le domaine ecclésiastique.

Cet essai fera apparaître que l'interprétation miltonienne de l'Écriture ne semble en rien prédisposer l'auteur au républicanisme. Long à se déclarer, celui-ci se définit d'abord négativement: il vaut mieux parler d'anti-absolutisme. Ce n'est qu'en 1660, acculé par le péril de la Restauration, que John Milton, opportuniste, plus pragmatique. s'appuie sur l'Écriture pour exposer clairement son projet en faveur d'une "libre république" ("a free Commonwealth") - ou, selon son expression. "a frugal and self-governing democratie."

1. Cet article s'appuie sur la dernière partie ma thèse, "Théologie et politique dans l'œuvre en prose de John Milton," U de Clermont-Ferrand II, juin 1996.

2. Toutes les références se feront à The Complete Prose Works of John Milton, ed. Don Marion Wolfe, 8 vols. (New Haven: Yale UP, 1953-82). Voir Proposalls of (Certaine Expedients for the Preventing of a Civill War Now Feard, and the Settling of a Firme Government [20 October - 26 December 1659] 7: 336].

3. Voir la seconde édition de The Readie and Easie Way io Establish a Free ('ommonwealth (7: 427), par allusion au style de vie des foumis dans Prov. 6. 6-8: "the ant ... having no guide, overseer, or ruler" (Readie and Easie Way. 1" ed., 7: 362). Pour les citations bibliques, se reporter à la King James Version, 1611 (London: The British and Foreign Bible Society, 1975). 
La logique scripturaire de Milton. l'idée fondamentale que les lois divine ou humaines doivent se plier au bien de l'homme (Tetrachordon 2: 588) ne l'amenait pas à rejeter le régime monarchique au profit d'une république populaire. Quatre raisons en rendent compte.

Tout d'abord, il appert que Milton n'est pas anti-royaliste par nature. Il faut dire qu'avant 1646. avec les Niveleurs. il n'y a pas de républicains déclarés dans le pays d'Albion. Dans (Of Reformation Touching ChurchDiscipline in England (1641), il profère l'idéal d'une constitution mixte, c'est-à-dire qu'il accepte pour l'Angleterre du XVIle siècle l'idée polybienne du gouvernement équilibré (Histoire 6: 2.10): "[where] under a free, and untutor'd Monarch, the noblest, worthiest and most prudent men, with full approbation, and suffrage of the People, have in their power the supreame, and finall determination of highest Affaires" (Reformation 1: 599).

Tout le monde, sauf les laudiens, l'aurait applaudi; y compris Charles Ier, lequel, dans sa Réponse aux XIX Propositions le 18 juin 1642, parle de la constitution anglaise comme d'une "monarchie réglée" - mais pouvait-il renier dans une déclaration publique ce qui était depuis fort longtemps la doctrine officielle? L'élément monarchique corrige les maux de l'aristocratie [division, faction] et ceux de la démocratie [tumultes, violence, licencel: réciproquement, la démocratie et l'aristocratie neutralisent les risques de débordement autoritaire du principe monarchique: la Chambre des communes, parce qu'elle seule vote la levée de l'impôt. parce qu'elle est compétente pour la mise en accusation des agents du roi s'ils enfreignent la loi ("a king can do no wrong"; voir Psaumes 94.20); la Chambre des lords, parce qu'elle a le pouvoir judiciaire - "an excellent screen between the prince and people." ${ }^{4}$ Les royalistes, encore moins Milton, ne croient pas au pouvoir absolu du roi: il est lié par la loi divine ou naturelle; par son serment du couronnement, le roi s'est engagé à préserver les lois quas vulgus elegerit, celles que le Parlement aura votées - à défaut de quoi il se parjure: "all Christian soverainty is by law, and to no other end but to the maintenance of the common good," écrit le polémiste dans An Apology against a Pamphlet (1: 946). En 1649, une fois devenu républicain, il précise sa pensée pour reprocher à Charles Ier, dans Eikonoklastes, son droit de veto, synonyme d'arbitraire: "he took upon him to have a negative will, as the transcendant and ultimate Law

4. Bernard Cottret, Michel Lemosse, and Michael Hearn, Anthologie de la civilisation britannique (Paris: Bréal, 1993) 73-74. Voir Glenn Burgess, Absolute Monarchy and the Stuart Constitution (New Haven: Yale UP, 1996). 
above all our Laws: and to rule us forcibly by Laws to which we our selves did not consent. but complain'd of" (3: 573).

L'absolutisme royal pose que le roi est non seulement legibus solutus, mais encore absque limitibus (Digeste 1: 4.1: "quod principi legis habet vigorem"). La souveraineté du roi le place au-dessus des lois: son pouvoir est illimité. On identifie la majestas à la prérogative royale [sa potestas extraordinaria \& libera]. Or, celle-ci est prévue par la common law, laquelle, de l'aveu de Milton, prime sur les dispositions parlementaires [!]. Surtout. le droit divin des rois ne posait que son irrésistibilité: il n'accordait pas de pouvoirs spécifiques au roi. Il servait aussi à condamner l'idée d'une autorité séculière indépendante dans l'Église: la pyramide épiscopale s'élevait jusqu'au roi. chef de l'Ecclesia anglicana. d'où la devise "No bishop, no king."

Plus intéressant, Milton voit l'organisation politique [roi - Parlement sujets] comme la réplique du schéma théologique [Dieu - Christ l'humanité]. Si Dieu est souverain, Il ne peut rien faire à l'encontre du droit, de la justice: Il est lié par sa promesse: en second lieu, l'homme a chuté de son plein gré: il jouissait du libre arbitre: enfin. Dieu a livré son fils, Christ, pour qu'il soit notre libérateur. Pour Milton. la suprématie rovale est réelle, mais elle ne saurait exister s'il n'y avait pas la liberté des sujets: ${ }^{5}$ le roi est lié par la loi. Et le Parlement, "our deliverers" (The Doctrine \& Discipline of Divorce 2: 226), n'est-il pas le lieu où les sujets dialoguent avec le roi, comme Christ est le Temple où le crovant communie avec Dieu? Dans An Apology. le libelliste clame que le Parlement est plus qu'un médiateur de la vie politique: "God by manifest signes and testimonies sets them as the mediators of his cov'nant[.] which he offers us to renew" (1: 927).

Ensuite, dans The Reason of Church-Governement Urg'd against Prelaty. pour vérifier que le presbytérianisme s'accommode du régime monarchique, alors que d'autres avaient allégué que l'Église presbytérienne "s'accord[ait] aussi bien avec la monarchie que Dieu avec le diable," Milton affirme qu'il ne fait aucun doute que le roi est l'oint de Dieu (1: 859: I Sam. 24.10: 26.11: "the LORD's anointed"): or, poser le jure divino des rois sur la base de l'Épître aux Romains 13.1 ("Tout pouvoir vient de

5. "Monarchy is made up of two parts, the Liberty of the subject, and the supremacie of the King" (Of Reformation 1: 592).

6. Jacques Ier, lors de la conférence de Hampton Court (14 janvier 1604). 
Dieu"). dans la logique de ses adversaires, revenait à nier le droit des peuples.

Deux ans plus tard. un passage de la préface à la seconde édition de The Doctrine \& Discipline of Divorce apparaîtrait comme une sérieuse mise en garde au Parlement si les arguments développés ne faisaient partie d'un raisonnement: "to resist the highest Magistrate though tyrannizing. God gave us never expresse allowance. only he gave us reason, charity, nature and good example to bear us out" (2: 229). Le Parlement s'oppose au divorce alors que celui-ci est cautionné par une loi divine; en revanche, il résiste au roi, ce à quoi Dieu ne l'autorise pas expressément.

Dans son plaidoyer pour l'abolition de la censure, il faisait peu de cas de la défense des privilèges parlementaires (2: 559-60), leur préférant la liberté d'expression. Il aura changé d'avis en 1649 , bien qu'il dise qu'il faut se soumettre à l'autorité légitime, celle des rois ou des magistrats.

Certes, l'analogie invite à rapprocher la répudiation de l'épouse odieuse de la destitution d'un mauvais roi. mais il ne s'agit pas d'abolir l'institution. ${ }^{7}$ Il n'y a qu'un glissement de sens: de lien extérieur formel, le mariage devient l'amour conjugal; la royauté, elle, échappe à la personne pour s'exprimer dans le règne hors partage de la loi.

Si Milton revendique le sacerdoce universel. la prêtrise royale ne pouvait englober les hommes indifféremment, mais seulement "les purs" (Tit. 1.5: voir Areopagitica): sa haine du vulgaire. des gens aspirant à une liberté déshonnête, son ressentiment pour leurs revendications déraisonnables l'empêchent d'envisager un républicanisme démocratique. Sa haute conception de la nature humaine, où il invite l'homme, image de Dieu, a fortiori s'il est chrétien, à prendre conscience de sa dignité, de sa liberté, de sa responsabilité, l'amène à formuler un élitisme incompatible avec "le gouvernement du peuple" - d'un régime politique où la souveraineté reviendrait à l'ensemble des citoyens.

S'ensuit une longue période de silence, de 1645 à la parution de The Tenure of Kings and Magistrates (13 février 1649). Milton se méfie-t-il? Le 21 avril 1647, dans une lettre à Carlo Dati, il fait part de ses soucis matériels: il doit prendre garde à sa vie, sauver ses biens (Works 2: 764) n'a-t-il pas épousé la fille d'un squire royaliste? S'il consigne, dès 1637-38, dans son Commonplace Book, que les peuples libres haïssent le nom de roi

7. Pierre du Moulin (Regii sanguinis clamor ad coelum, adversus parricidas anglicanos [La Hague, 1652]) écrit: "récemment [Milton] est passé de la dislocation du mariage à la dissolution des royaumes" (Works 4: 1051). 
(1: 440), suivant là Sulpice Sévère, ${ }^{8}$ il se refuse à prendre position pour l'une des deux parties. espérant la conciliation - dès lors que les parasites de la Cour auront été châtiés.

Ce n'est qu'une fois qu'est scellé le destin de Charles ler qu'il fait irruption sur la scène politique pour condamner vigoureusement les presbytériens. Ces derniers s'en étaient pris violemment à sa théoric du divorce: Milton avait là l'occasion d'assouvir sa vengeance, de dénoncer leur duplicité. Cet écrit lui valut le poste de secrétaire des Affaires étrangères. Commissionné pour faire voler en éclats l'image du roi (Eikon Basilikè), il défend la république a contrario. Il ne réprouve pas la royauté, mais "la Monarchie tyrannique" (Eikonoklastes 3: 388), "celle où [le Prince] foulant aux pieds les loix de nature, abuse de la liberté des francs sujects, comme de ses esclaves, et des biens d'autruy, comme des siens": " elle correspond à la $\pi \alpha \mu \beta \alpha \sigma \imath \lambda \varepsilon \iota \alpha$. . système où le roi exerce un pouvoir incontrôlé en vue de son propre intérêt (Pro populo anglicano defensio [1651], 4: 477-78). En 1654, dans un passage autobiographique de la Seconde défense du peuple anglais, il révèle qu'il a écrit "ce qu'il est permis en général de faire à un tyran" (4: 626).

De 1655 à 1659 , il ne dit plus rien. Tandis que son pays se prépare à accueillir Charles II, Milton est le dernier à faire entendre sa voix pour sauver "la bonne vieille cause." Là encore, il précise que la royauté, à condition qu'elle soit élective, n'est pas un mauvais régime en soi: seulement les circonstances particulières de l'Angleterre de 1660 interdisaient son retour: "monarchy of it self may be convenient to some nations, yet to us who have thrown it out, received back again, it cannot but prove pernicious" (Ready and Easie Way 7: 377-78; 449).

C'est là que son engagement pour un régime républicain s'exprime ouvertement, qu'il prend sa véritable dimension. Le danger imminent de la Restauration le pousse à faire des propositions concrètes. Quelles solutions suggère-t-il? Est-il possible de les considérer comme assises sur la Bible, norme discriminatoire absolue?

8. Sulpice Sévère, Sacrae historiae (Leyden, 1635) 1.56.

9. Comparez: "what is properly his own guilt, not imputed any more to his evil Counsellors (a Ceremony us'd longer by the Parlament then he himself desir'd) shall be laid heer without circumlocutions at his owne dore" (Eikonoklastes 3: 341).

10. Jean Bodin, Les six livres de la République 2: 55.

11. Aristote, Politique 4: 10. 4. 
C'est alors qu'il donne une définition positive d'"une libre République." Dans sa Politique, Aristote avait défini l'État comme la réunion de familles, de plusieurs villages, en vue de mener la meilleure vie possible (7: 8. 4). Cette collectivité devait se suffire pour les besoins quotidiens (3: 9.12). La cité existe pour le bonheur des hommes [eudaimonia], leur beauté morale. Il ne fait guère de doute. dans Eikonoklastes. que la définition miltonienne est aristotélicienne: "every Common-wealth is in general defin'd. a societie sufficient of it selfe. in all things conducible to well being and commodious life" (3: 458).

Elle appelait deux précisions: d'abord, pour Milton. l'autarcie allait de pair avec la liberté des hommes. Selon Aristote, il n'existe que deux sortes de politie: la démocratie, "quand le pouvoir est détenu par une majorité d'hommes de naissance libre, mais pauvres" (Politique 4: 4.6); l'oligarchie, "quand il appartient à une minorité d'hommes riches d'origine plus noble"; ensuite, le publiciste voyait dans sa communauté politique utopique le règne de la raison publique, qu'incarne le Rump, "Conseil suprême général de la nation" (Observations upon the Articles of Peace 3: 348), qu'il opposait à la volonté privée du roi, que celle-ci se manifeste par sa voix négative, par le contrôle de la milice, ou par la perception des subsides.

On objectera que Dieu a institué la royauté: dans le Premier Livre de Samuel 8 , Il accède à la requête des Israélites, lesquels voulaient un roi [a captain], non seulement afin qu'il juge, mais encore pour qu'il les conduise à la guerre (8.20). Pour Milton, dans Readie and Easie Way, il est évident que Dieu, parce qu'Il s'estime rejeté, leur accorde un roi malgré Lui: "God in much displeasure gave a king to the Israelites, and imputed it a sin to them that they sought one" $(7: 359 ; 424) .^{12}$

Il en aura la ferme conviction de 1649 - avec Tenure (3: 202-3) - à 1660, dans Brief Notes upon a Late Sermon (7: 476). Dieu savait par avance que Son peuple allait demander un roi. L'ire divine s'explique parce que les Israélites avaient défié Sa volonté: "ils ne veulent plus que je règne sur eux" (1 Sam. 8.7). Certes, ils reprochaient la mauvaise administration des deux fils de Samuel [Yoël, Aviya], auxquels le prophète, devenu vieux, avait délégué une partie de ses attributions: dévoyés par le lucre, s'adonnant à la corruption, ils avaient fait dévier le droit. Bien que la demande des Israélites Lui déplût, qu'Il essayât de les dissuader par la voix

12. Milton, Church-Govemement: "It is to be fear'd [God permitted this insurrection of Episcopacy] in his wrath, as he gave the Israelites a King" (1: 781); Defensio prima: "[T]hose headstrong people found themselves accused for having sought [a king]" (4: 353-54: voir 347, 369-70); voir Tenure 3: 207-08. 
de son prophète (vous pleurerez à cause du roi que vous vous serez choisi, [1 Sam. 8.19]), Dieu dit à Samuel d'écouter ce qu'ils lui diraient. Un passage du Deutéronome laissait prévoir l'instauration de la royauté: lorsque vous serez installés en Canaan. "thou shalt say. I will set a king over me. like all the nations that are about me" (Deut. 17.14).

L'auteur la présente comme une réponse à la requête du peuple, avec la réserve qu'il ne pouvait faire roi que l'homme désigné par Dieu. Le paragraphe exprime une réticence à l'égard de la royauté, parce que le roi voudra la puissance [posséder un grand nombre de chevaux], parce qu'il convoitera les richesses ["neither shall he multiply to himself gold and silver"]. parce qu'il sera orgueilleux [trop de femmes dévoient son cœur!]. Pour remédier à cela. le Deutéronome stipule que le roi fera une copie de la Loi pour veiller à ce qu'elle soit mise en pratique. mais aussi pour qu'il ne s'élève pas au-dessus de ses frères. ni qu'il s'écarte du commandement. Aussi Samuel ne saurait-il exposer les droits régaliens, mais il dénonce les usages de la plupart des rois (1 Sam. 8.11-18): il avertit le peuple d'Israël d'un grave danger (Defensio prima 4: 351-53).

C'est comme si le peuple anglais choisissait de retourner en Égypte, dans "la maison de servitude" (Exod. 20.2), s'écrie le pamphlétaire, stupéfait (Readie and Easie Way 7: 387, 462). Essayons d'éclaircir ce qu'il a voulu dire.

L'enjeu de l'exode - la sortie d'Égypte, suivie de la marche dans le désert - est la liberté au service de Dieu: il ne s'agit pas de secouer le joug de l'esclavage du Pharaon pour une liberté anarchique. L'alliance sinaïtique devait codifier la liberté d'Israël; dans la mesure où sa morale est réponse à une initiative absolument gratuite de Dieu, l'obéissance n'est pas une source de mérites, mais une reconnaissance de ce que l'on doit à Dieu: "if thou will obey my voice indeed, and keep my covenant, then ye shall be a peculiar treasure unto me above all people" (Exod. 19.5).

"And ye shall be unto me a kingdom of priests, and an holy nation" (Exod. 19.6: voir 1 Pierre 2.5). Tout ce que le Seigneur a dit, le peuple, unanime, s'engageait à l'appliquer. Uni à Dieu, Israël ne recevrait que des bénédictions. Or, il allait bientôt s'avérer incapable de respecter son engagement, ainsi que le vérifie sa demande d'un roi. Semblablement, Dieu avait élu l'Angleterre, le nouvel Israël; Il avait délivré les Anglais de l'esclavage de Charles Ier: à l'inverse des Israélites, ils avaient rejeté leur roi pour faire de Dieu "our leader and supreme governor" (Tenure 3: 236). Ayant déposé, c'est-à-dire lapidé politiquement, son roi, l'Angleterre pouvait espérer l'assistance divine en 1649 avec l'inauguration d'une ère 
nouvelle: dans Eikonoklastes, elle devait prendre garde à ce que Dieu ne la délivre plus si elle s'obstinait à revenir en arrière (3: 580). Aujourd'hui, elle ne savait pas gré à Dieu de ce qu'Il l'avait secourue. Le Très-Haut, pour punir son ingratitude ("our ingratefull backsliding" [Readie and Easie Way 1 7: 358]), pourrait retirer sa grâce, laisser l'Angleterre à son sinistre sort.

Cela signifie que pour garder l'alliance que Dieu a conclue avec la nation, l'Angleterre doit rompre définitivement son alliance avec le roi, car "nul homme ne peut servir deux maîtres," se plaît à dire le polémiste, suivant là Matthieu 6.24. ${ }^{13} \mathrm{Ce}$ "covenant" avec Dieu, de plus, est conforme à la loi de nature, car les magistrats ne sont que mandatés par le peuple, lequel garde virtuellement le pouvoir (Tenure 3: 202) - sinon l'homme se réduirait à une bête brute pour accepter d'être gouverné par la volonté d'un de ses semblables.

Si Dieu avait inféré de leur insoumission que les Juifs composaient "un peuple à la nuque raide" (Exod. 32.9), les Anglais, eux, par leur obstination à vouloir courber l'échine devant un roi héréditaire, avéraient leur stupidité; plus encore, selon Readie and Easie Way 2, ils niaient leur liberté naturelle: "[no] Nation, styling themselves free, can suffer any man to pretend hereditarie right over them as this lord" (7:427).

Ce caractèré contraire à la loi de nature secondaire a pour corollaire que le peuple aura été fait pour le roi - inversion de l'ordre des choses: salus populi suprema lex. C'est une perversion également religieuse: le roi doit être vénéré comme un demi-dieu (7: 425: Readie and Easie Way 1 7: 360), ce que Milton rend par l'idée de luxe, voire de prostitution. C'est qu'il faut $y$ voir une infidélité à Dieu - n'a-t-Il pas créé les hommes naturellement libres? - ou à Christ, seul $\mathrm{Roi}^{1+4}$ - ne les a-t-il pas rachetés (Readie and Easie Way 2 7: 463)? Il y aurait là une véritable idolâtrie civile. Se rendre esclave d'un seul homme, débauché par surcroît, est aussi inhumain qu'anti-chrétien.

Toutefois, si Dieu reproche aux siens leur endurcissement, il poursuit fidèlement en leur faveur son plan de salut. La question que Milton a dû se poser en voyant son pays en plein désarroi en 1659-60 est bien celle-ci: quelle est la volonté de Dieu dans "les insanités civiles" que vit

13. Milton, Readie and Easie Way 1: "we could not serve two contrary maisters, God and the king" (7: 411).

14. Milton, Readie and Easie Way 1: "Christ, our common King and Lord" (7: 364, 429). 
l'Angleterre? ? $^{\text {t5 }}$ Veut-Il l'éprouver? Il est encore possible qu'Il fasse surgir "les enfants de la liberté renaissante," les héritiers de la promesse faite à Abraham, mais l'Angleterre, par "une folie épidémique," n'a-t-elle pas irrémédiablement rejeté la grâce divine? Auquel cas, la Restauration serait un fléau de Dieu pour châtier les Anglais de leur obstination. ${ }^{16}$ On rejoignait la logique royaliste: le tyran est voulu par Dieu pour punir le peuple méchant.

Il est deux points positifs: Dieu a voulu que les hommes puissent librement choisir leur forme de gouvernement: en outre, Il avait un $a$ priori favorable pour une république aristocratique, "gouvernement formé des gens absolument les meilleurs par leur vertu."17 Il aurait désiré le maintien du régime des juges. Quel était-il? En fait, lorsqu'il parle de la république juive, Milton fait l'amalgame de plusieurs éléments.

Il renvoie d'abord à la plus haute autorité civile de l'État amphictyonique. Avant qu'Israël ne s'installe en Canaan, il y aurait eu douze $n^{e} s \hat{\imath}$ 'îm, "des gens de renom dans la communauté, les princes de la Tribu de leurs ancêtres" (Nombres 1.16). Ces délégués ou responsables des douze מטה (Exod. 16.22) se réunissaient autour du Sanctuaire central pour prendre des décisions communes. Or, il n'est pas question, à l'époque. d'un véritable gouvernement central ni d'une machinerie administrative régie par une autorité souveraine: "En ces jourslà. chacun faisait ce qui lui plaisait" (Juges 21.25). Outre les dirigeants ou porte-parole des Tribus, il y avait l'assemblée du peuple, la kol-q hal. ${ }^{18}$ Tout israélite mâle de plus de vingt ans pouvait y prendre part, mais les anciens $\left[z^{e} q e n i m\right]$, ses membres les plus importants en raison de ce qu'ils étaient sagement éprouvés, devaient la constituer: dans l'acception hébraïque, ils définissaient l'ecclesia au sens où l'ensemble serait présent dans ses parties les plus caractéristiques. ${ }^{19}$ Ces notables, Milton les verrait bien dans "un Sénat des hommes les plus en vue" ("principal" [Readie and Easie Way 2 7: 439]).

15. Lettre to Henry Oldenburg, 20 December 1659 (Works 7: 515).

16. Milton, Readie and Easie Way 2: "Much less will he hear us when we cry herafter, [because] once deliverd by him from a King, insensible and unworthie of [his] high mercies [we] are returning precipitantly, if he withhold us not, back to the captivitie from whence he freed us" (7: 449-50).

17. "aristox" (Aristote, Politique 4: 7. 2).

18. "all the congregation of the children of Israel" (Nombres 26.2).

19. Chez Milton, le "peuple" désigne les citoyens les meilleurs. 
Avec la sédentarisation en Canaan, les communautés urbaines devinrent les unités politiques de base. On retrouvait l'assemblée publique au niveau de la ville (Ruth 3.11: "the city of my people"), avec les anciens, chefs de famille ou de clan, pour rendre leurs verdicts. Ce que Milton aurait souhaité pour son pays: "every countie in the land [shall be] made a kinde of subordinate Commonaltie" (Readie and Easie Way 7: 383, 458).

Ces magistrats judiciaires avaient été établis par Moïse: sur les conseils de son beau-père, afin d'alléger son fardeau, il avait relevé parmi les anciens "des hommes de valeur, craignant Dieu, dignes de confiance, incorruptibles" pour en faire des chefs de milliers, de centaines, de cinquantaines ou de dizaines (Exod. 18.13-26): ils devaient juger le peuple en permanence dans les affaires peu importantes (Deut. 1.9-18) pour laisser l'arbitrage des différends plus graves à la charge du patriarche. Ce corps, Nombres 11.16 le réduisait à soixante-dix âmes, les plus puissants ayant accaparé l'autorité. Et Milton de l'assimiler au grand Sanhédrin, gerousia (1 Macc. 4.44) ou presbuterion (Luc 22.66), "Conseil des Anciens." que présidait le grand-prêtre ${ }^{20}$ - bien que son institution date du retour de l'exil babylonien, en dépit de ce que les soixante-dix Anciens n'avaient aucune fonction de gouvernement. Néanmoins, il s'agit là d'une élite bien pensante. Milton ne désire rien d'autre: les plus méritants doivent avoir des postes de commandement, administrer la justice, au niveau local, régional, mais aussi national - les hommes de savoir seront "les piliers inébranlables de l'État" (Of Education 2: 398).

Ces juges doivent être distingués des šoptîm [juges], vaillants guerriers, chefs charismatiques que Dieu suscite, qu'Il pénètre de Son esprit, pour qu'ils délivrent Son peuple des assaillants: Otniel. Éhoud, Baraq, Gédéon, Samson en étaient, Samuel aussi. Or, Milton, avec Dieu, déplore l'éviction de Samuel: il aurait voulu que l'on continuât à déléguer l'exécutif à un personnage haut en couleurs, ${ }^{21}$ d'autant qu'en plus de

20. Milton, Readie and Easie Way 2: "Therefore among the Jews, the supream Councel of seaventie, call'd the Sanhedrim, founded by Moses, consisted of members chosen for term of life" (7: 370-71, 436).

21. Dans The Present Means and Brief Delineation of a Ffree Commonwealth [23 February - 4 March 1660], si Milton invite le Général George Monck à réunir à Londres "the chief Gentlemen" (7: 393) pour les convaincre d'organiser des élections, il ne voit pas d'inconvénient, dans sa réplique au sermon de Matthew Griffitth sur Prov. 24.21, à ce qu'il soit intronisé: "yet chusing out of our own number one who hath best aided the people, and merited against tyrannie, the space of a raign or two we may chance to live happily anough, or tolerably" (Brief Notes upon a late Sermon 7: 482). 
conduire les armées, Samuel avait gouverné Israël - comme Débora, Tola, Yaïr, Jephté, Elôn ou Abdôn avant lui.

Cette infrastructure politique, il conviendrait de la dresser dans l'Angleterre agonisante de 1660. Milton est d'avis qu'il faut reprendre "le gouvernement des Juges" tel qu'il figure dans l'Ancien Testament. La constitution miltonienne, si elle est dans l'esprit de l'Ancien Testament, puisqu'il s'agit d'établir une "juriscratie" (sauf que le gouvernement du droit, de la raison publique, se substituerait à Dieu, encore qu'll fût la loi suprême ou la recta ratio), s'inspire vaguement du gouvernement prémonarchique. Si le schéma miltonien plaide pour une décentralisation de la justice dans des Cités-États autonomes, il opte pour un Conseil de quatre cents membres, élu par les comités locaux, lesquels administreraient les villes, bourgs ou comtés: il choisirait un organe exécutif composé de sept à vingt-et-un membres. Ce dernier n'a pas son équivalent dans l'Ancien Testament: d'autre part, loin de songer à "la dictature des Juges," Milton pense à "the mild Aristocracy of elective Dukes, and heads of Tribes joyn'd with them" (Of Reformation 1: 575), par opposition à "the easie, or hard-handed Monarchy's." Cette douce aristocratie assurerait l'intérim jusqu'à l'avènement de "thy universal and milde Monarchy" - la royauté de Christ (616).

Il serait difficile de ne pas rapprocher les propos de Milton du projet de James Harrington dans Oceana (1656). d'autant qu'il y fait allusion l'ouvrage fut publié en deux éditions distinctes (l'une mentionne: "imprimé par J[ohn] Streater, pour Livewell Chapman" [19 September], l'autre sera pour le libraire Daniel Pakeman). Tandis que Milton veut "une libre République," Harrington souhaite "une République égale."22 Toute leur différence est là. L'auteur d'Oceana appelait à reproduire le système de la Ligue des Douze Tribus (Reuben, Siméon, Juda, Issachar, Zabulon, Benjamin, Dan, Nephtali, Gad, Asher, Manassé, Ephraïm): il ne doutait nullement qu'elle eût existé. Elle comprenait deux sortes d'assemblée.

"Tout le peuple," l'Ecclesia Dei, "[t]he Church or Congregation of the People of Israel assembl'd in a military manner" (49; Juges 20.2),

22. C'est-à-dire "a Government establish'd upon an equal agrarian, arising into the Superstructures or Three orders, [working] by an equal Rotation thro the suffrage of the People given by the Ballot"; "The center or Fundamental Laws are, first. the Agrarian. proportion'd at two thousand Pounds a year, and stating Property in Land at such a balance. That the Power can never swerve out of the hands of the Many. Secondly, the Ballot . . an equal Election or Rotation" (James Harrington, Oceana and Other Works, ed. John Toland [London, 1787] 55, 204). 
constituait la législature: il ne s'agissait pas des fantassins [quatre cent mille hommes]. mais des chefs [captains] - des milliers. etc.. soit vingtquatre mille personnes élues pour un mois. Ce corps pouvait également choisir les magistrats.

Soixante-dix Anciens, nommés par Dieu, mais élus par le peuple, composaient le Sénat (Deut. 1.13); il siégeait à la Cour du Tabernacle. Ce sanhédrin était chargé d'exécuter la loi: "[its] function consisted in the administration of the law made." 23

$\mathrm{Ce}$ à quoi il fallait ajouter les juges de la municipalité - "Courts consisting of three and twenty Elders sitting in the Gates of every City" (Deut. 16.18), soit environ six mille hommes. Chaque village ou presque disposait en outre d'un Triumvirat de juges.

Ce type de schéma est irrecevable pour Milton par le fait qu'il commande une assemblée populaire - de mille membres par exemple, comme le suggère Harrington: dès lors, le Sanhédrin aurait un rôle subordonné. Serait-il une simple cour d'appel: "a standing Judicatory of Appeal from the Courts in the Gates, thro[ugh]out the Tribes" (The Art of Lawgiving, 1659)? S'il faisait des propositions de loi, l'Ancien Testament fait apparaitre que celles-ci devaient encore être ratifiées par l'assemblée pour devenir effectives. Enfin, pour le polémiste, l'expérience prouvait que "les démocraties populaires" n'avaient rien apporté de bien au peuple, si elles ne le l'avaient pas ruiné (Readie and Easie Way 2 7: 438-39); il repoussait le renouvellement annuel du Sénat, la rotation partielle, parce qu'elle avait trop d'affinité avec la roue de la fortune (435).

Alors Milton se penchait sur l'Évangile: dix-huit ans plus tôt, il voulait que l'on calque l'organisme ecclésial sur le système politique en vigueur (Of Reformation 1: 600): là, il semble d'avis que l'État reproduise le modèle de l'Église, le schéma presbytérien, où les pasteurs, assistés de diacres, élus par la congrégation, gouvernent la communauté de croyants, l'Église concrète. Cet organigramme ne saurait accepter qu'un seul roi, Christ: son royaume est à venir. Le controversiste insiste sur le fait que le Fils de Dieu a pris la condition d'un servus afin que l'homme soit affranchi: s'il a montré l'exemple aux "grands" [les gouvernants], ${ }^{2-}$ Christ

23. Harrington 51 .

24. "[T]hey who are greatest, are perpetual servants and drudges to the publick at their own cost and charges, [and] neglect their own affairs; yet live soberly in their 
a affilié les hommes à la divinité: il leur a conféré une entière liberté, $y$ compris politique, puisqu'il destine son intimation dans la Première Épître aux Corinthiens 7.23 ("be not ye the servants of men") aux hommes comme tels, dans leur réalité purement humaine, non religieuse - "au prix de sa propre servitude, il a assis [leur] liberté politique sur une base solide" (Defensio prima 4: 375).

Christ réitère le rejet de la royauté dans l'Évangile: lorsque les fils de Zébédée viennent le voir pour lui demander de participer au jugement eschatologique, de siéger à sa droite ou à sa gauche, il leur répond que "si quelqu'un veut être grand, qu'il soit votre serviteur" (Luc 22.25-7). C'est que ses disciples, par opposition à ce que font "les princes des Gentils," ne doivent pas chercher à dominer: ${ }^{25}$ "a free Commonwealth [is] planely commended or rather enjoind by our Saviour himself, to all Christians, not without remarkable disallowance and the brand of Gentilism upon kingship" (Readie and Easie Way 7: 359, 424). C'est là que le bât blesse, car le sacerdoce de l'ensemble des croyants appelle la démocratie. Dans Defensio secunda, Milton affirme que si l'on instaure l'égalité dans l'Église, l'on aura la discipline évangélique, mais qu'en revanche, au cas où elle s'applique à l'État, elle sera "le gouvernement du peuple" (4: 633).

L'État républicain, qu'il voit assis sur la loi vétérotestamentaire, s'accorde conceptuellement avec la discipline ecclésiale voulue par Christ, "le gouvernement des assemblées" (Of Reformation 1: 606): les anciens, dit l'Évangile, seront "irréprochables" (Tit. 1.6); assistés de diacres, ils devront guider les fidèles sur le droit chemin. Il est indéniable qu'ils formaient une élite morale. Cela, Harrington, dans Oceana, ne pouvait le concevoir: les Apôtres auraient emprunté le nom d'ecclesia pour désigner les Églises ramassées ["gathered churches"] afin d'avérer qu'ils voulaient que le gouvernement ecclésiastique fût démocratique (49): "the Apostles, to shew they intended the Government of the Church to be popular, ordain'd Elders . . . by the holding up of hands (or, free Suffrage of the People) in very Congregation or Ecclesia." 26

Harrington prétend que les presbytériens le rendaient aristocratique en s'appuyant sur l'ordination des soixante-dix; or, les Anciens avaient d'abord été désignés par le peuple, avant d'être consacrés par Moïse: dans

families, walk the streets as other men, may be spoken to freely, familiarly, friendly" (Readie and Easie Way 7: 360, 425).

25. Milton, Defensio prima: "one cannot wish to dominate and remain a Christian"

(4: 379). Voir Readie and Easie Way 7: 364, 429; Marc 10.42-45; Matt. 20.25-28.

26. Harrington 137. 
The Art of Lawgiving (405), il écrit que chacune des douze tribus proposait six candidats. inscrivant leurs noms sur des rouleaux, qu'elle remettait à Moïse: celui-ci préparait deux urnes, l'une contenant les soixante-douze noms ainsi obtenus. l'autre avec soixante-dix bulletins portant la mention "elder," plus deux bulletins vierges (ils seront tirés par deux hommes. "l'un s'appelait Eldad, l'autre Médad" [Nombres 11.26]). Harrington est persuadé qu'élection ("Take you wise men, and understanding, and known among your tribes") et ordination ("and I will make them rulers over you" [Deut. 1.13]) ne faisaient qu'une: "[the people] ever after substituted their Successors by Ordination. [a] Ceremony most usually perform'd by imposition of hands. [whence] derives that which was introduc'd by the Apostles in the Christian Church. "27

Milton, lorsqu'il fait l'exégèse de la Première Épître à Timothée 4.14 ("Neglect not the gift which was given thee by prophecy, and by laying on the hands of the presbytery") dans Animadversions, ne rejoint pas Harrington: il insiste sur l'article défini devant $\pi \rho \varepsilon \sigma \beta \nu \tau \varepsilon p l o v$ pour dire que l'accent n'est plus mis sur l'ordination par un seul homme, l'évêque, mais sur le fait que Timothée, lequel n'est en aucun point comparable à un évêque ordinaire, a été consacré à son ministère par le conseil des anciens: ${ }^{28}$ - non pas par l'ensemble des fideles. Cette ordination, d'autre part, bien que cérémonie, n'a rien d'un sacrement: "it would be improper to call ordination $\chi \alpha$ ópı $\sigma \mu \alpha$, when as it is rather onely $\chi \varepsilon\llcorner\rho \iota \sigma \mu \alpha$, an outward testimony of approbation" (709).

Le projet miltonien, s'il se veut fidèlc au régime prémonarchique de l'Ancien Testament, devait reproduire le modèle ecclésial tel que le prosateur le lit dans le Nouveau Testament. À la base de la discipline presbytérienne, le conseil paroissial, composé de pasteurs. d'anciens [elders], élus par l'ensemble des fidèles pour administrer les affaires de la communauté concrète établie en un lieu: ils sont flanqués de diacres, chargés de secourir les pauvres ou d'assister les malades. Cette structure, allant du classis, avec, à un échelon supérieur, le synode régional, jusqu'au sommet, l'assemblée générale, sera adoptée le 29 août 1648. Elle subit quelques altérations chez Milton, puisque le publiciste élimine les assemblées intermédiaires pour ne garder que "le Consistoire paroissial" ainsi que le synode national (Church-Governement 1: 789). En soulignant la dignité du chrétien, sa liberté, son adoption, il devait croire au sacerdoce

27. Harrington 137.

28. Voir Milton, Church-Govemement 1: 707-09; 767-68; Of Prelatical Episcopacy 1: $650-51$. 
universel des croyants, en l'autarcie des communautés religieuses. Son ecclésiologie. il la fondait sur "[a] holy and equall Aristocracy" (Of Reformation 1: 600), un presbytérianisme libéral, pendant naturel de "la douce Aristocratie" politique (Of Reformation 1: 575).

Milton fustige la vaine gloire des prélats - orgueil, avarice, ambition. Cette prétention à se faire valoir, notamment par les cérémonies, va à l'encontre de l'Évangile. lequel stipule que l'Église devra se conduire avec simplicité, qu'elle sera faible dans le monde, vile, digne de mépris. Par là, elle réalisera le dessein de Dieu exprimé dans la Première Épître aux Corinthiens 1.27-28 - confondre ce qui est fort, réduire à néant ce qui existe aux yeux des hommes: "he sent Foolishnes to confute Wisdom, Weaknes to bind Strength, Despisednes to vanquish Pride" (ChurchGovernement 1: 824).

La fatuité épiscopalienne vexe l'Évangile, pour lequel, dans Tite 1.7, l'épiscope ne sera pas arrogant ("selfwilled"; "Dieu s'oppose aux orgueilleux, mais aux humbles il accorde sa grâce" [1 Pierre 5.5]). L'élévation des évêques jusqu'à une hauteur insolente est inversement proportionnelle à l'avilissement de Christ dans l'Épître aux Philippiens: "Et il a pris la condition d'un serviteur" (2.7).

"[C]hose altière," l'épiscopalisme s'opposait à la connaissance de Dieu, à la foi (De doctrina christiana 6: 128). Il était injustifié que les hommes d'Église soient pourvus d'une juridiction de for externe (2 Cor. 10.4-5). Pour Milton, les prélats sont les voleurs ou brigands de la parabole du berger - Dieu ne leur avait remis aucune mission; aussi ne pouvaient-ils entrer par la porte, c'est-à-dire Christ, dans l'enclos des brebis; ils prétendaient par leurs propres moyens apporter la connaissance des choses célestes. À moins qu'ils ne fussent des mercenaires, prêts à sacrifier les brebis à leur intérêt, en les livrant à la mort; ils voulaient dominer à leur seul profit; Christ, lui, s'était dessaisi de sa vie pour qu'elles vivent (1 Jean 10.1-18). Ils n'avaient d'yeux que pour "Mamôn et leur Ventre" (Of Reformation 1: 577). Le polémiste compare les dignitaires ecclésiastiques à des loups ("ravenous and savage wolves" [Church-Governement 1: 856; Actes 20.29]), lesquels s'infiltrent dans la bergerie pour dévorer le cheptel. En dehors de l'imposition de la croyance ou des pratiques, il a en vue les cours diocésaines, où l'on est entraîné de force pour payer quelque amende. Or, la juridiction de l'Église ne comprend que la censure ecclésiastique, avec ses deux instruments, "la Réprimande \& le Blâme" (846). Ces intrus, brigands ou mercenaires, conclut Milton, il conviendrait de les chasser de 
l'Église de Dieu (voir Considerations Touching the Likeliest Means to Remove Hirelings out of the Church [1659]).

Les conclusions miltoniennes plaident en faveur d'un rapprochement de son républicanisme avec sa réforme ecclésiale à plusieurs égards: tout comme il souhaite "une Discipline douce et humble" (Of Reformation 1: 613) pour l'Église. "[f]eed the flock of God which is among you, taking the oversight thereof, not by constraint, but willingly; not for filthy lucre, but of a ready mind; Neither as being lords over God's heritage, but as being examples to the flock" (1 Pierre 5.2-3). ${ }^{29}$

Milton veut un régime politique apte à servir les administrés: les dirigeants, "many industrious equals" (Readie and Easie Way 2 7: 427), seront les serviteurs du peuple, comme les presbytres servent les fidèles. La prédication de la Parole, ainsi que l'office de "gardien" du peuple de Dieu, ressortissent au presbytère: ils ne pouvaient appartenir à un seul homme placé au-dessus de ses frères, leur imposant sa foi (l'on ne saurait considérer Pierre comme un "super-évêque"). Semblablement, les magistrats civils ne devraient pas s'enorgueillir devant les leurs - le roi, lui, se considérait comme un demi-dieu; il voulait dispenser $s a$ propre loi. Les épiscopes doivent instruire, exhorter ou reprendre les fidèles (2 Tim. 4.2): les Sénateurs doivent engager le peuple sur le chemin de la vertu. Si l'Église ne pouvait exister en dehors de la Loi de Dieu, mais que celle-ci prône la liberté religieuse, la société civile ne saurait se concevoir en dehors des lois: la liberté civique repose sur les lois humaines. ${ }^{30}$

Tout comme il a choisi un système de congrégations locales autogérées par les chrétiens les plus dignes pour l'Église de Christ, Milton opte pour une fédération de comtés, pluralité d'États dans l'État, dirigés par les hommes les plus compétents: chacun d'eux œuvrerait pour lui-même, mais ils seraient unis dans la différence; ensemble, ils s'efforceraient de vivre harmonieusement. Son sénat suprême se limiterait à diriger la politique extérieure (Readie and Easie Way 2 7: 443); il aurait un pouvoir limité, car les comités locaux, éventuellement regroupés "en des assemblées plus générales," auraient un œil critique sur ce qu'il entreprendrait: "they may declare and publish their assent or dissent by deputies within a time limited

29. Citation favorite de Milton. Les rétërences à 1 Pierre 5.2-3 sont récurrentes dans Church-Governement 1: 760, 783, 791, 838, 856.

30. Harrington: "[T]he Liberty of a Commonwealth consists in the Empire of her Laws" (45). 
sent to the Grand Councel; yet so as their judgment shall submitt to the greater number of other commonalties" (7: 459).

Tout le monde a la possibilité d'être fait ministre à condition que Dieu ait mis des dons en lui (De doctrina christiana 6: 570-71): "[h]e that inabl'd with gifts from God, and the lawfull and Primitive choyce of the Church assembl'd in convenient number, faithfully feeds his Parochiall Flock, is a true Apostolick Bishop" (Of Reformation 1: 537).

Parallèlement, les membres du sénat, une minorité vertueuse, sur la voie de la régénération, élue par un peuple redéfini comme "la classe moyenne," dirigeraient le Commonwealth miltonien. Si la confusion politique ambiante s'explique parce que les Anglais s'apprêtaient à "réadmettre" la monarchie, cela prouve que les voix de la minorité, soucieuse de liberté dans son hostilité à la restauration, devraient prévaloir: le polémiste écarte la loi du plus grand nombre, car il n'est pas juste, ni raisonnable, écrit-il dans Readie and Easie Way 2, que les opposants à la fin principale du gouvernement asservissent le nombre moindre vertueux (7: 455), parce qu'il revendique son bien le plus précieux, le sang de Christ, par lequel il a reconquis sa liberté. Les quelques-uns à exceller en prudence, dans la pratique des affaires, en industrie, comme en force morale, seront forcément majoritaires: ils doivent l'emporter dans les suffrages, où qu'ils soient organisés (Defensio secunda 4: 636). Il est logique que le cadre institutionnel privilégie les hommes ayant le plus de mérite: faut-il les priver de l'usage de la liberté parce que "le plus grand nombre" en abuse? La lex naturalis rapporte les lois, sources du droit, ainsi que le gouvernement civil, au salut des citoyens les meilleurs. ${ }^{31}$ Cette minorité que Milton appelle de ses vœux à prendre les rênes du pouvoir ne serait-elle pas "le reste de Son peuple" religieux (Ésaïe 11.16)? Le pamphlétaire parle du Rump de 1659-60 comme étant le reste des braves du Long Parlement épuré fin 1648. Ces individus d'exception sont des justes, des hommes que l'Esprit illumine; guidés vers Dieu, suivant librement la loi de nature originaire, ils connaissent le bien.

Le républicanisme de Milton s'accorde excellemment avec sa vision de la liberté, car il est d'avis, dans The History of Britain [1672], que seuls

31. Milton, Defensio prima 4: 533; "They who seek nothing but thir own just libertie, have alwaies right to winn it and to keep it, when ever they have power, be the voices never so numerous that oppose it" (Readie and Easie Way $27: 455$ ). 
les hommes bons seraient en droit de l'obtenir ("For libertie hath a sharp and double edge sword fitt onelie to be handl'd by just and vertuous men" [5: 449: Héb. 4.12]), mais sa logique n'est pas humaine: l'aspect judiciaire qu'il fait revêtir à son conseil d'État ne s'accommode guère de l'idée d'un gouvernement - doux serviteur du peuple. Si la liberté véritable est "là où est l'Esprit du Seigneur" (2 Cor. 3.17), Milton ne veut pas d'une république théocratique, car le pouvoir civil doit rester séparé du glaive spirituel.

Son républicanisme est l'aboutissement d'une logique politique contradictoire: si Milton est en faveur du "gouvernement des meilleurs," une élite morale pourvue de la liberté chrétienne, il doit rejeter le "gouvernement d'un seul." à savoir la monarchie hćréditaire. au nom de ce que les hommes naissent libres et égaux; il devait également rejeter la démocratie, autrement dit le gouvernement de la majorité, car la liberté n'est pas licence; l'égalité débouche sur des injustices.

En revanche, il est dans la logique de la théologie évangélique, que Milton a bien vue: "many are called, but few are chosen," dit Matthieu 22.14 (voir De doctrina christiana 6: 460). Cette poignée de privilégiés a son équivalent dans l'ancienne dispensation: le reste.

Milton avait réussi à ancrer sa libre république dans l'organisation ecclésiale voulue par Christ; il était parvenu à la faire émerger du système politique élaboré par Moïse. Cependant, il n'avait pas su aller jusqu'au bout de sa logique: si l'ecclésiologie dans le Nouveau Testament prépare la monarchie christique, l'État républicain mosaïque s'inscrit comme gouvernement prémonarchique dans l'Ancien Testament. Quelle royauté la théocratie juive annonce-t-elle? Celle de Christ: Saül ne préfigure-t-il pas le Sauveur? 